\title{
Osteoid osteoma of the third metatarsal bone: A case report
}

\author{
By Dae Gon Moon ${ }^{1}$, Kwang Woo Nam ${ }^{3}$, Chang Lim Hyun ${ }^{2}$, Kyu Bum Seo ${ }^{3, *}$ \\ ${ }^{1}$ Jeju National University School of Medicine \\ ${ }^{2}$ Department of Pathology, Jeju National University School of Medicine, \\ ${ }^{3}$ Department of Orthopedic Surgery, Jeju National University School of Medicine, Jeju, Korea
}

\begin{abstract}
Osteoid osteoma is a benign bone tumor composed of osteoid and atypical bone. The most commonly known sites of following tumor are long tubular bones, especially femur and tibia. Yet, development of osteoid osteoma in the foot is unusual, and there aren't much reported literatures of metatarsal osteoid osteoma. In this article, we report a case of the osteoid osteoma in the 3rd metatarsal bone of a 14-year-old male patient.
\end{abstract}

Key words: Osteoid osteoma, Foot, Metatarsal

\section{INTRODUCTION}

Osteoid osteoma is a benign, osteoblastic tumor comprised of osteoid and atypical bone, and was first described by Jaffe. ${ }^{1)}$ The most common sites of origin for this tumor are long tubular bones, especially femur and tibia, with presentation of pain that responds well to non-steroidal anti-inflammatory medications. Yet, osteoid osteoma occurring in the foot is unusual and accounts for approximately $4 \%$ of cases, ${ }^{2)}$ and there aren't much reported literatures of metatarsal osteoid osteoma. ${ }^{2)}$ Osteoid osteoma developed in the metatarsal bone may be difficult to diagnose due to its rarity, unless the surgeon maintains a high index of clinical suspicion.

Hence, we report a case of fourteen-year-old boy who developed third metatarsal osteoid osteoma, with focus on the clinical history, radiographic appearances, histopathologic findings and adequate treatment.

\section{CASE REPORT}

A fourteen-year-old boy was presented in our outpa-

Received: May 17, 2018; Revised: June 20, 2018; Accepted: June 20, 2018

*Correspondence to : Kyu Bum Seo

Department of Orthopedic Surgery, Jeju National University School of

Medicine, 15, Aran 13-gil, Jeju 63241, Republic of Korea

Tel: 82-64-717-2709, Fax: 82-64-717-1131

E-mail: cbnuoskbs@jejunu.ac.kr tient clinic with five months history of painful swelling over the dorsal aspect of the left forefoot. No definite history of trauma was noted and persistence and intensity of symptoms kept the patient from participating in sports activities. The pain was aggravated at night and relieved by non-steroidal anti-inflammatory drugs.

Physical examination revealed no clinical abnormality except for the tenderness and swelling over the third metatarsal shaft of the left forefoot. The results of complete blood count, C-reactive protein level and serum chemistries, were all within normal limits. Radiographic evaluation, including anteroposterior and oblique views of the foot, demonstrated a small radiolucent area in mid-diaphysis area of the third metatarsal bone, surrounded by reactive sclerosis (Fig. 1A and 1B). Axial computed tomography of the third metatarsal bone showed enlarged metatarsal containing low attenuation nidus with intrinsic calcification and surrounding sclerosis (Fig. 2). A clinical history and imaging findings were consistent with osteoid osteoma.

As for surgical intervention, longitudinal incision on the lateral side of the third metatarsal was used for exposure. With guidance of $\mathrm{K}$-wire under $\mathrm{C}$-arm image intensifiers, and the lesion and surrounding bone were surgically excised using rongeur to match the contour of the original metatarsal (Fig. 3A and 3B). Alleviation of patient's pain was noticed immediate postoperatively. To prevent stress fractures after removal of osteoid osteoma, 

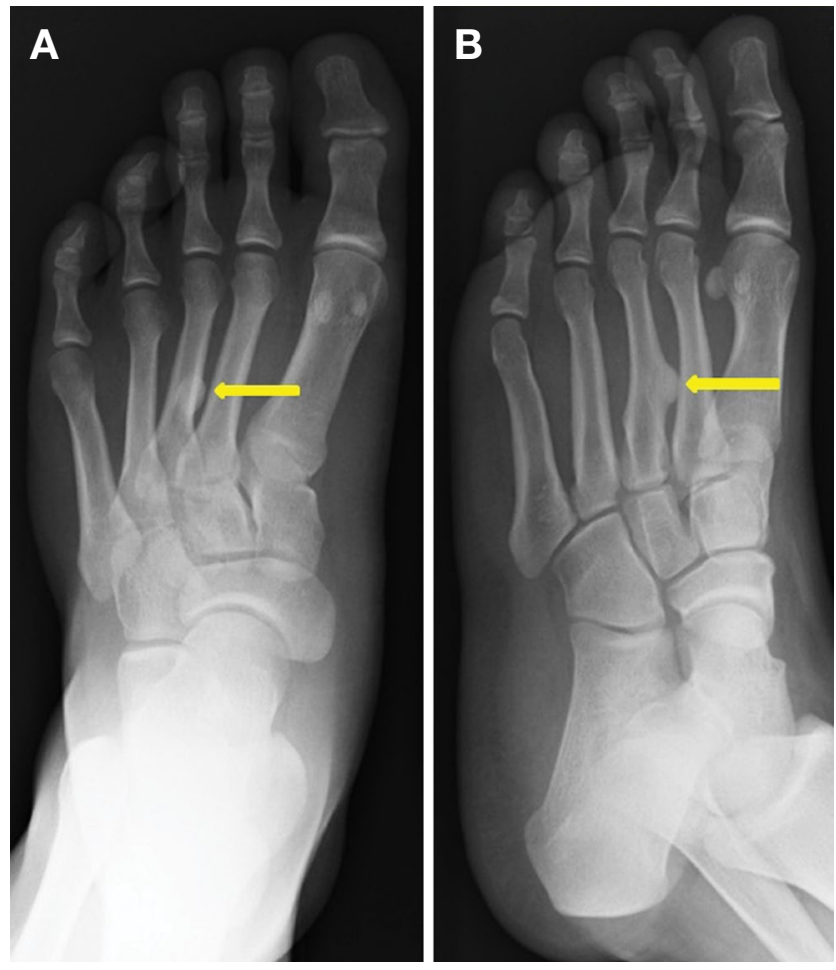

Figure 1. Anteroposterior and oblique radiographs of the left foot, showing a small radiolucent area in the protruded lesion with surrounding reactive sclerosis in the mid-diaphysis area of the third metatarsal bone (Yellow arrow).

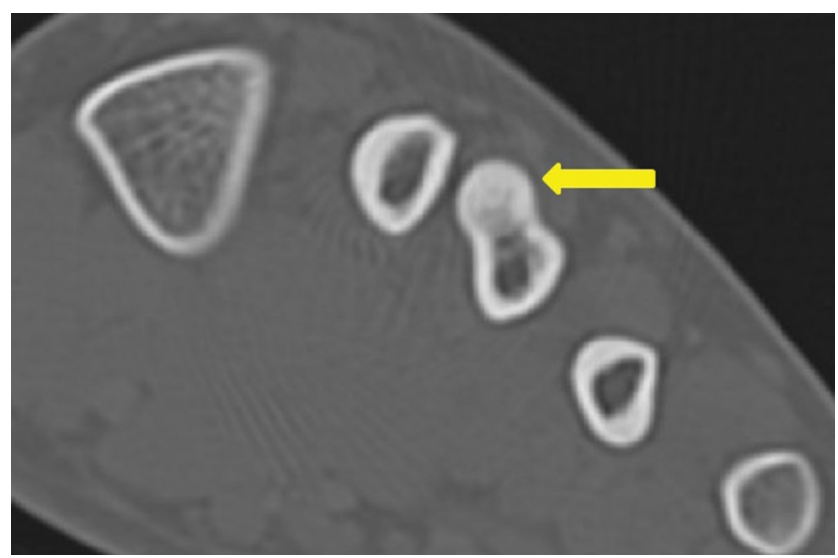

Figure 2. Axial computed tomography of the third metatarsal bone showed enlarged metatarsal, low attenuation nidus with intrinsic calcification and surrounding sclerosis (Yellow arrow).

splinting was performed for 2 weeks, with additional application of removable orthosis for 2 weeks. Patient was advised not to participate in sports activities for 2 months postoperatively. Histopathologic examination revealed osteoid osteoma (Fig. 4). The patient remained asymp-
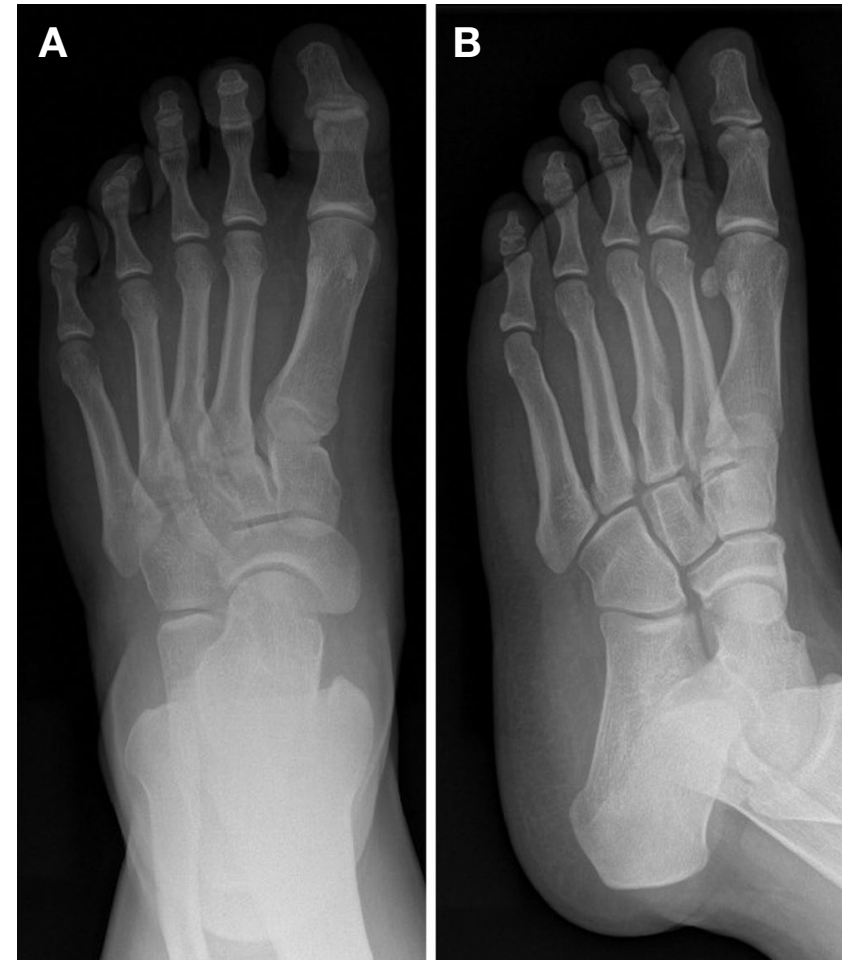

Figure 3. Anteroposteror and oblique radiographs of the left foot, showing surgically excised the lesion and surrounding bone in the mid-diaphysis area of the third metatarsal bone.

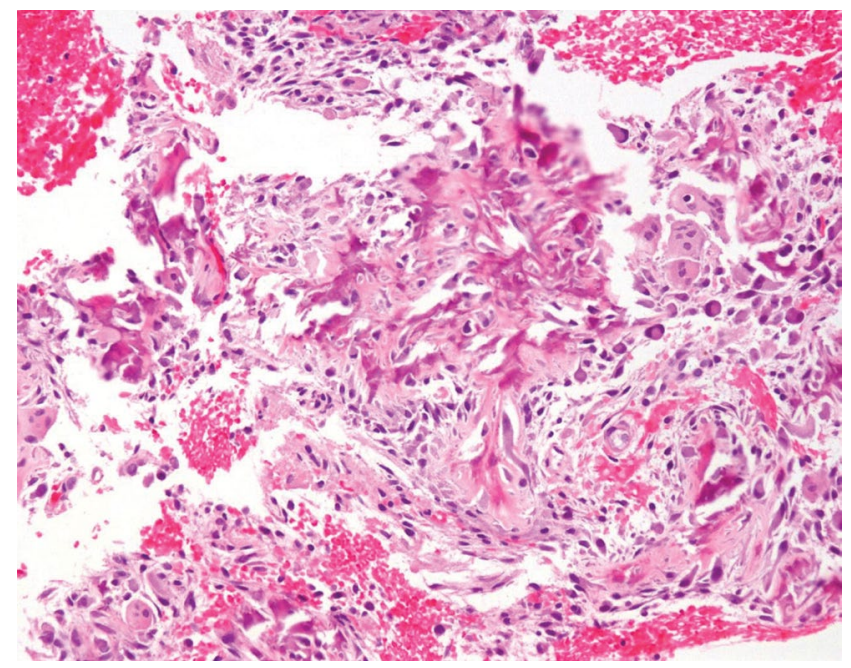

Figure 4. The central nidus of tumor demonstrates interconnected trabeculae of woven bone and osteoid. The osteoid is microscopically disposed in a sheet-like configuration and organized into microtrabecular arrays. The intertrabecular space is filled with fibrovascular stroma (hematoxylin and eosin stain; original magnification, $\times 200$ ).

tomatic, with no evidence of local recurrence till 1 year postoperatively. 
The study submitted has been performed in accordance with the ethical standards of institutional and/or national research committee and reviewed by IRB. After reviewing submitted IRB protocol and other related materials, the participated IRB members upon "Approval" of the research.

\section{DISCUSSION}

A distinct benign osteoblastic tumor composed of osteoid and woven bone, osteoid osteoma was first described by Jaffe. ${ }^{1)}$ It is a common lesion that accounts for $10 \sim 12 \%$ of all benign bone tumors. Osteoid osteoma is usually presented in children and young adults (range: $10 \sim 25$ years old), with a male to female ratio $>2: 1{ }^{3)}$ $50 \%$ of osteoid osteoma appears in the diaphyseal and metaphyseal regions of long tubular bones, especially the femur and tibia. ${ }^{2-4)}$ In the small bones of the foot, it accounts for approximately $4 \%$ of cases $^{2)}$ and the most commonly known site is talus. ${ }^{5)}$ Osteoid osteoma usually accompany 1 10 mm sized vascular nidus, with presentation of variable amount of peripheral sclerotic reaction. ${ }^{6}$

Osteoid osteoma often shows symptom of progressive night pain that is temporarily relieved by aspirin or NSAID. The mechanism of pain relief by anti-inflammatory medication is that the level of prostaglandin is significantly increased in the nidus, which is the presumable cause of pain and vasodilatation. ${ }^{4}$

Other physical findings vary with the site of the tumor. Clubbing is common when tumor affects distal phalanx of fingers or toes. Decrease of range of motion and adjacent muscle atrophy may be present if lesion is located within the joint. Growth disturbance can occur in immature skeleton. ${ }^{4,7)}$

Typical radiographic appearance of a central nidus with surrounding bony sclerosis is the crucial hint in diagnostic image studies. However, there are some osteoid osteomas with lucent nidus without any sclerosis on plain radiograph or CT scan which are difficult to identify and may delay the diagnosis. ${ }^{8)}$ Some of chronic ankle pain developed by ankle impingement, ankle sprain, os trigonum syndrome, or inflammatory arthropathy is hard to differentiate from pain caused by osteoid osteoma in the foot.

Osteoid osteoma occurred in the foot usually presents in cancellous bone or within a joint, where the periosteal reaction is minimal, as opposed of its usual site of intracortical lesion of long bone, associated with prominent solid periosteal reaction. ${ }^{9)}$ Thus, identification of osteoid osteoma in the midfoot can be challenging if lack of periosteal reaction and complex radio-anatomy is combined. In the patient described in this article, osteoid osteoma was located in subcortical lesion, displayed small radiolucency with peripheral sclerosis, but no periosteal reaction.

The radiologic differential diagnosis of osteoid osteoma arising in the foot includes chondromyxoid fibroma, osteochondroma, chondroblastoma, enchondroma, and osteoblastoma. ${ }^{4,7)}$ Differentiation from these entities usually is clear by closely examining clinical symptoms and radiographic findings. For example, osteoblastoma differs from osteoid osteoma in that it is more progressive with higher growth potential $>2 \mathrm{~cm}$ in diameter, and it frequently lacks nocturnal pain. ${ }^{10)}$ Definite diagnosis can be established by histopathologic evaluation.

Microscopic examination reveals nidus of osteoid, surrounded by osteoid trabeculae that displayed varying degrees of mineralization in a background of vascular fibrous connective tissue. A variable number of multinucleated giant cells and well-differentiated osteoblasts can be seen. The perinidal osteosclerotic zone usually consists of well-mineralized bone with dense cortical bone in cortical osteoid osteoma, thickened trabecular bone in cancellous osteoid osteoma and abundant localized periosteal new bone formation in periosteal osteoid osteoma. ${ }^{3,7)}$ Our patient had dense cortical bone in the perinidal osteosclerotic zone with well-mineralized bone.

Surgical excision of nidus of osteoid osteoma is crucial, for it provides immediate and permanent relief of symptoms. Incomplete excision likely to lead to recurrence., ${ }^{3,4,7)}$ In our patient, alleviation of symptom was achieved through surgical excision of nidus, and no evidence of recurrence of the tumor was noted after 12 months of postoperative follow up.

\section{CONCLUSION}

Osteoid osteoma developed in metatarsal bone is relatively rare. A detailed clinical history with a high index suspicion is required for accurate diagnosis. For immediate and permanent remission of symptoms without recurrence, adequate surgical excision of the tumor lesion is essential. 


\section{REFERENCES}

1. Jaffe HL. Osteoid osteoma: a benign osteoblastic tumor composed of osteoid and atypical bone. Arch Surg 1935;31:709-28.

2. Shereff MJ, Cullivan WT, Johnson KA. Osteoid-osteoma of the foot. J Bone Joint Surg Am 1983;65:638-41.

3. Wu KK. Osteoid osteoma of the foot. J Foot Ankle Surg 1991;30: 190-4.

4. La Croix ML, Thomas JR, Nicholas RW. Subperiosteal osteoid osteoma of the distal phalanx of the fourth toe. Orthopaedics 2001;24:695-6.

5. Snow SW, Sobel M, DiCarlo EF, Thompson FM, Deland JT. Chronic ankle pain caused by osteoid osteoma of the neck of the talus. Foot Ankle Int 1997;18:98-101.

6. Greenspan A. Benign bone-forming lesions: osteoma, osteoid osteoma, and osteoblastoma. Clinical, imaging, pathologic, and differential considerations. Skeletal Radiol 1993;22:485-500.

7. Barca F, Acciaro AL, Recchioni MD. Osteoid osteoma of the phalanx: enlargement of the toe-two case reports. Foot Ankle Int 1998; 19:388-93.

8. Mommert I, Heuschmidt M, Suckel A. Intraarticular osteoid osteoma as a cause of chronic ankle pain. Orthopade 2009;38:269-73.

9. Shukla S, Clarke AW, Saifuddin A. Imaging features of foot osteoid osteoma. Skeletal Radiol 2010;39:683-9.

10. Temple HT, Mizel MS, Murphey MD, Sweet DE. Osteoblastoma of the foot and ankle. Foot Ankle Int 1998;19:698-704. 